Proceedings of the 45th International School and Conference on the Physics of Semiconductors "Jaszowiec" 2016, Szczyrk

\title{
Investigation of Localized Electric Field in the Type-II InAs/GaAsSb/GaAs Structure
}

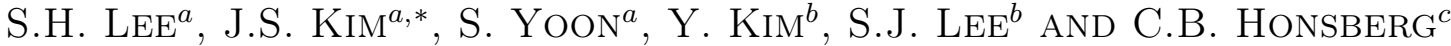 \\ ${ }^{a}$ Department of Physics, Yeungnam University, Gyeongsan 712-749, Korea \\ ${ }^{b}$ Division of Convergence Technology, Korea Research Institute of Standards and Science, Daejeon 305-340, Korea \\ ${ }^{c}$ School of Electrical, Computer and Energy Engineering, Arizona State University, Tempe, Arizona 85287, USA
}

The effect of localized electric field $(F)$ was investigated in the type-II InAs/GaAsSb/GaAs structures. To compare type-I to type-II, two types of samples with different Sb contents was grown by molecular beam epitaxy, whose Sb contents are 3\% (type-I) and 16\% (type-II), respectively. In the both samples, we performed excitation power dependent-photoreflectance at $10 \mathrm{~K}$ and the result showed that the period of the Franz-Keldysh oscillation, revealed above the band gap $\left(E_{g}\right)$ of GaAs, was broadened in the only type-II system, which means that $F$ was also increased because it is proportional to the period of the Franz-Keldysh oscillation while the period of the Franz-Keldysh oscillations stayed unchanged in type-I system. This phenomenon is explained by that the $F$ was affected by the band bending effect caused by the spatially separated photo-excited carriers in the interface between GaAsSb and GaAs. The $F$ changed linearly as a function of square root of excitation power as expected for the $F$. Moreover, $F$ was calculated using fast Fourier transform method for a qualitative analysis, which is in a good agreement with the theory of triangular well approximation.

DOI: 10.12693 /APhysPolA.130.1213

PACS/topics: 78.66.Fd, 78.40.-q, 73.40.Lq, 73.50.Pz

\section{Introduction}

The InAs/GaAsSb/GaAs heterostructure system has attracted much attention recently because of its potential application in semiconductor fields. Particularly, its type-II band alignment provides an excellent opportunity to improve the performance of both heterojunction bipolar transistors and optoelectronic devices due to their nature of the separation of photo-excited electrons and holes [1-4]. Therefore, InAs/GaAsSb/GaAs system is very attractive for both of academic interest and industrial application.

In this type-II system, optical properties have been studied in many groups using photoluminescence (PL) measurements $[5,6]$. It shows that a significant blue-shift of the peak energy occurs under a moderate excitation power, which can be well explained by the band bending effect due to the spatially separated photo-excited carrier in type-II band alignment. In addition, the separated charges by the band bending effect also induces localized electric field $(F)$. This $F$ can be calculated by using theory of approximate triangular well $[5,6]$. However, it is difficult and complicated to obtain the value of $F$ experimentally. Therefore, the $F$ has not been researched anywhere even though this $F$ is intriguing to many researchers for academic interest. In this work, we present a specific investigation of the effect of localized electric field in type-II system and experimentally obtain the value of $F$ using photoreflectance (PR) spectroscopy.

\footnotetext{
*corresponding author; e-mail: jongsukim@ynu.ac.kr
}

\section{Experimental details}

The samples were grown by a solid-source molecular beam epitaxy (MBE) system on a semi-insulating GaAs (001) substrate. As shown in Fig. 1, the structure consisted of a $400 \mathrm{~nm}$ undoped GaAs buffer layer, $\approx 120 \mathrm{~nm} \mathrm{InAs/GaAsSb} / \mathrm{GaAs}$ layers and $20 \mathrm{~nm}$ cap layer. To grow sub-monolayer quantum dot (SML-QD) stacks, eight periods of InAs/GaAsSb/GaAs layers were embedded. Each period of the SML-QD layers comprised of five cycles of $\operatorname{InAs}(0.5 \mathrm{ML}) / \mathrm{GaAsSb}(2.5 \mathrm{ML})$

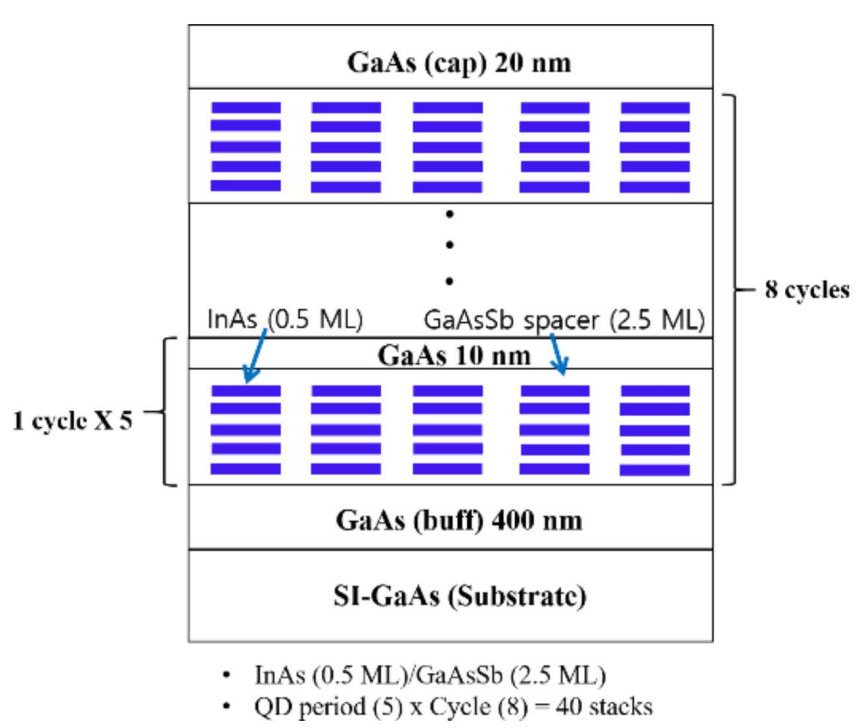

Fig. 1. Schematic sample of InAs/GaAsSb/GaAs SML-QD. 
and it was capped with $10 \mathrm{~nm}$ of GaAs layer. The Sb content of the GaAsSb spacers was 3\% (type-I) and $16 \%$ (type-II), respectively. To investigate the effect of localized electric field $(F)$, we performed excitation power dependent-photoreflectance (EPPR) at $10 \mathrm{~K}$ to enhance the localized effect of photo-excited carriers. A He-Ne laser $(632.8 \mathrm{~nm})$ is used as an excitation beam at various $I_{\mathrm{ex}}(0.075-21 \mathrm{~mW})$. In this technique, a mechanically chopped laser beam $(400 \mathrm{~Hz})$ modulates the interface electric fields of the sample by generating electron-hole pairs. The probe light from a tungsten-halogen lamp passing through a monochromator illuminated the sample surface. The reflected light was detected by a Si photo detector. The more detailed PR setup was described in Refs. [7, 8].

\section{Results and discussions}

Figure 2 shows the photoreflectance (PR) spectra for type-I (black solid line) and II (red solid line) at $10 \mathrm{~K}$ with $\mathrm{He}-\mathrm{Ne}$ laser of $0.075 \mathrm{~mW}$. The PR spectra contain features assigned to the GaAs band-to-band $\left(E_{g}\right)$, InAs/GaAsSb quantum dot (QD) transition, GaAsSb spin-orbit (SO) transition [9], and GaAs SO signal. The Franz-Keldysh oscillation (FKO) was also observed. The period of FKO is known to be strongly related to the built-in electric field of semiconductors and their heterostructures. The electric field is proportional to the period of FKO [10]. However, in this structure, we cannot expect any built-in electric field because these structures are composed of only undoped layers. Nevertheless, FKOs were revealed in both structures due to the appearance of the interface states as a consequence of $\mathrm{Sb}$ absorption at the interface and/or the possible interchanging reaction between the $\mathrm{Sb}$ atoms and the As atoms $[11,12]$.

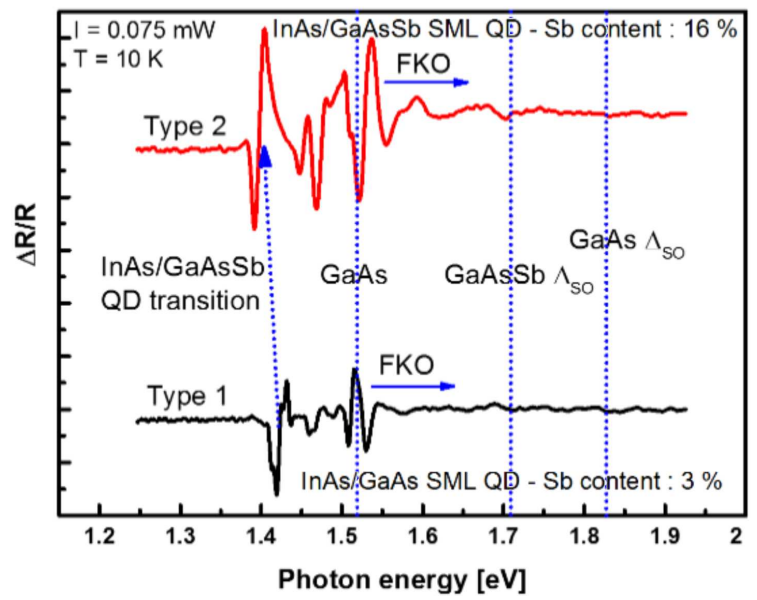

Fig. 2. PR spectra at $10 \mathrm{~K}$ for type-I (Sb 3\%) and type-II (Sb 16\%).

Indeed, the effect of $F$ at the InAs/GaAsSb interface may be more intriguing to all researchers in this field. However, it is so complicated to analyse the localized effect in the SML-QD structures. Therefore, we are currently interested in the effect of $F$ at the interface between GaAsSb and GaAs layers.

To investigate the effect of localized electric field $(F)$ in InAs/GaAsSb/GaAs structure, we performed excitation power dependent-PR (EPPR) as shown in Fig. 3. By EPPR measurement, we can prove where the FKOs are originated from.

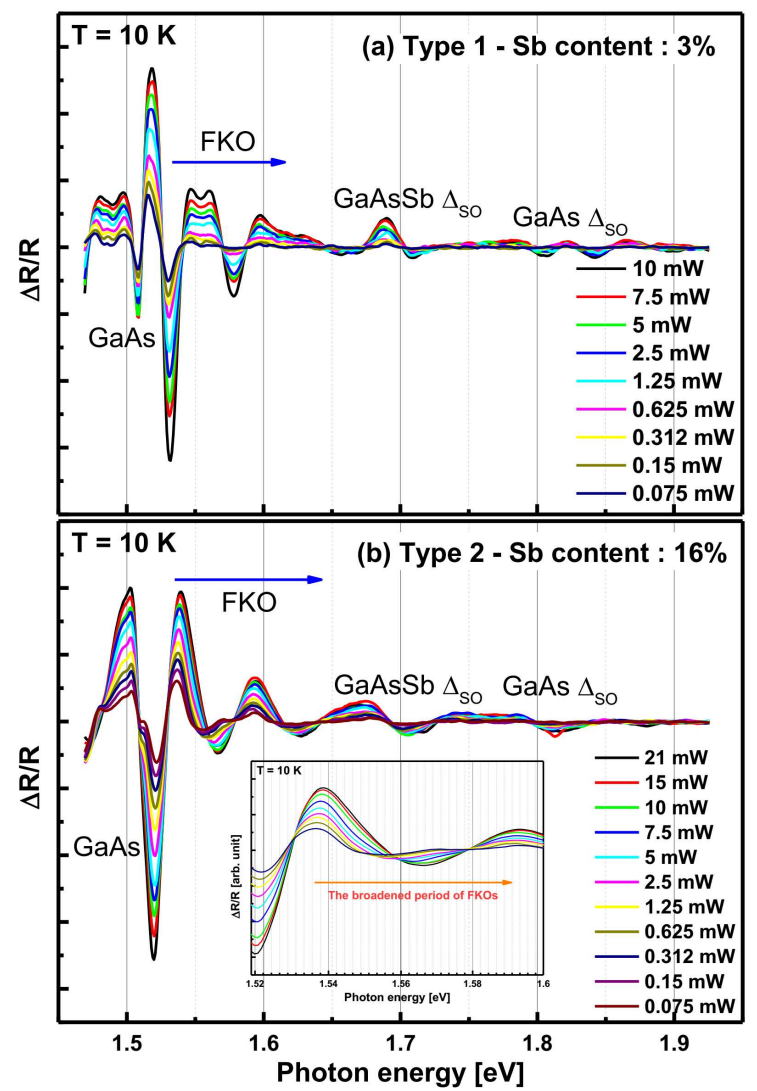

Fig. 3. EPPR spectra at $10 \mathrm{~K}$ for (a) type-I (Sb: $3 \%$ ) and (b) type-II (Sb: 16\%). $900 \mathrm{~nm}$ short pass filter (SPF) was used for reducing PL signal of InAs/GaAsSb SML-QD.

Figure 3a shows the spectra of EPPR measurement for type-I system. Above the GaAs $E_{g}$, unchanged FKOs periods indicate that the $F$ is not originated from spatially separated states, which means that $F$ did not change with increasing excitation power $\left(I_{\mathrm{ex}}\right)$ because confined carriers in type-I system cannot spatially be separated into electrons in GaAs states and hole in GaAsSb states. In contrast, Fig. 3b shows that for type-II system the periods of FKOs were broadened with increase of $I_{\mathrm{ex}}$. It means that the $F$ also increases because confined carriers induce the localized field due to the band bending effect caused by the spatially separated photo-excited carriers in type-II alignment [5, 6].

For a qualitative interpretation of $F$ in type-II, the frequency of FKO can be extracted by fast Fourier transform (FFT) method $[7,8]$. Using this frequency, 

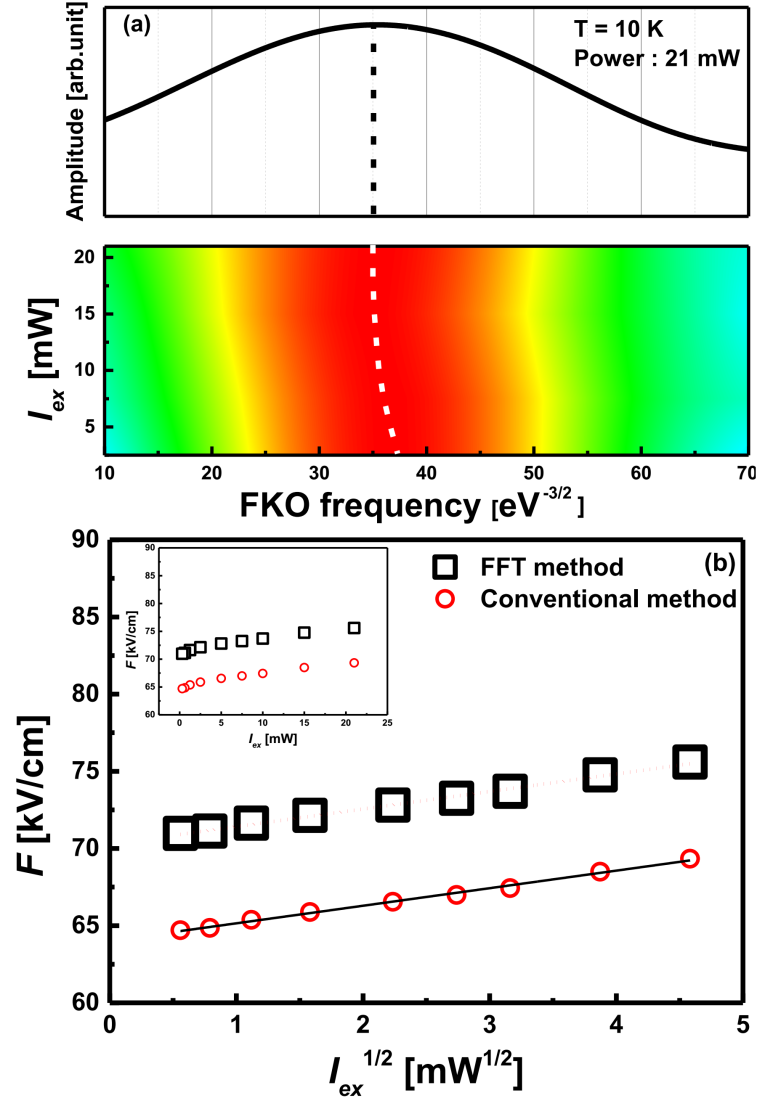

Fig. 4. (a) FKO frequency as a function of excitation power $\left(I_{\text {ex }}\right)$ and (b) localized electric field $(F)$ calculated from FKO frequencies as function of $I_{\mathrm{ex}}^{1 / 2}$ and the $F$ from FFT is compared with $F$ from conventional method.

we can calculate $F$ because the frequency of $\mathrm{FKO}$ is inversely proportional to electric field at an interface of a sample [7, 8].

Figure $4 \mathrm{a}$ shows contour plot of FKO frequencies spectra obtained by FFT with $I_{\mathrm{ex}}$. The frequencies of FKO were decreased with increase of $I_{\mathrm{ex}}$. The FFT results show broad spectra in the frequency domain. This broadness was caused by the broad electric field distributions in the interface of the sample from the unintentional diffusion of doping material at the doped interfaces and from overlapping of the electron-heavy hole and the electronlight hole transition frequencies components $[7,8]$.

Figure $4 \mathrm{~b}$ shows the value of $F$ calculated from the frequencies of FKO as a function of $I_{\mathrm{ex}}^{1 / 2}$, which is compared with $F$ calculated from conventional method. Both methods show deviation of only $\approx 5 \mathrm{kV} / \mathrm{cm}$ so that our results indicate the reliability of the experiments. In order to analyze the tendency of $F$, we consider the electric field induced by photo-excited carriers. The strong localized carriers near the interface form a charged plane and, correspondingly, produce an approximately triangular well with an electric-field strength of $[5,6]$ :

$$
F=\frac{2 \pi e n_{w}}{\epsilon_{0}} \propto I_{\mathrm{ex}}^{1 / 2} .
$$

Here, $n_{w}$ is the electron density generated in the thin layer region by an external photon of excitation power $I_{\text {ex }}$.

As seen in Eq. (1) mentioned above, $F$ is proportional to $I_{\mathrm{ex}}$ to the power of $1 / 2$. This result is in a good agreement with Eq. (1) derived by triangular well theory. If $F$ is at $\mathrm{GaAsSb} / \mathrm{GaAs}$ interface, the transition from holes states in GaAsSb to electron states in GaAs may be revealed in PR spectrum. In our PR spectrum in Fig. 2, the transition appears in photon energy ranging in 1.43$1.5 \mathrm{eV}$. However, we need to analyse those signals for further study because the oscillation in the range 1.43 $1.5 \mathrm{eV}$ is more complicated and overlapped with FKO of $\mathrm{GaAsSb}$ and the transition. In the future, we propose that the study of the oscillation will be performed so that a good work will be finished.

\section{Conclusions}

The effect of localized electric field $(F)$ was investigated in type-II InAs/GaAsSb/GaAs system by photoreflectance spectroscopy. EPPR was taken at $10 \mathrm{~K}$ to enhance carrier confinement effect in each quantum state. For type-I system, the period of the Franz-Keldysh oscillation was unchanged with increase of excitation power $\left(I_{\text {ex }}\right)$ because photo-excited carriers cannot spatially be separated, and thus do not induce $F$. On the contrary, for type-II system, type-II band alignment leads to the spatially separated photo-excited carriers. Therefore, the periods of FKO was broadened with increase of $I_{\text {ex }}$, which means that $F$ also increases due to the band bending effect. For qualitative analysis, the value of $F$ was calculated from the frequency of FKO extracted by FFT method. The calculated $F$ was proportional to $I_{\text {ex }}$ to the power of $1 / 2$ which is in a good agreement with Eq. (1) derived by triangular well theory.

\section{Acknowledgments}

This study was supported by the Priority Research Centers Program through the National Research Foundation of Korea (NRF) and funded by the Ministry of Education (2014R1A6A1031189). This study was supported by a 2013 Yeungnam University Research grant.

\section{References}

[1] H.Y. Liu, M.J. Steer, T.J. Badcock, D.J. Mowbray, M.S. Skolnick, F. Suarez, J.S. Ng, M. Hopkinson, J.P.R. David, J. Appl. Phys. 99, 046104 (2006).

[2] H.Y. Liu, M.J. Steer, T.J. Badcock, D.J. Mowbray, M.S. Skolnick, P. Navaretti, K.M. Groom, M. Hopkinson, R.A. Hogg, Appl. Phys. Lett. 86, 143108 (2005).

[3] J.M. Ripalda, D. Granados, Y. Gonzalez, A.M. Sanchez, S.I. Molina, J.M. Garcia, Appl. Phys. Lett. 87, 202108 (2005).

[4] K. Akahane, N. Yamamoto, S.-I. Gozu, N. Ohtani, Physica E 26, 395 (2005). 
[5] T.T. Chen, C.L. Cheng, Y.F. Chen, F.Y. Chang, H.H. Lin, C.-T. Wu, C.-H. Chen, Phys. Rev. B 75, 033310 (2007).

[6] Y.S. Chiu, M.H. Ya, W.S. Su, Y.F. Chen, J. Appl. Phys. 92, 5810 (2002).

[7] S.H. Lee, I.S. Han, C.W. Sohn, H.-J. Jo, J.S. Kim, S.J. Lee, S.K. Noh, J.O. Kim, Curr. Appl. Phys. 15, 1318 (2016).

[8] H. Mani, A. Joulie, A.M. Joulie, B. Girault, C. Alibert, J. Appl. Phys. 61, 2101 (1987).

[9] W.-H. Chang, T.M. Hsu, W.C. Lee, R.S. Chuang, J. Appl. Phys. 83, 7873 (1998).
[10] H.P. Hsu, P. Sitarek, Y.S. Huang, P.W. Liu, J.M. Lin, H.H. Lin, K.K. Tiong, J. Phys. Condens. Matter. 18, 5927 (2006).

[11] H.P. Hsu, P. Sitarek, Y.S. Huang, P.W. Liu, J.M. Lin, H.H. Lin, K.K. Tiong, Phys. Status Solidi A 204, 430 (2007).

[12] C.W. Sohn, I.S. Han, R.P. Smith, J.S. Kim, S.K. Noh, H. Choi, J.-Y. Leem, J. Korean Phys. Soc. 64, 1031 (2014). 